\title{
An Intelligent Wireless Modular System for Effective Disaster Management
}

\author{
${ }^{1} \mathrm{R}$ Bhakthavathsalam, ${ }^{2} \mathrm{~K}$ Vishruth, ${ }^{2}$ Likith Ponnanna P B, ${ }^{2}$ Deepak B, ${ }^{2} \mathrm{M}$ Kaushal and ${ }^{2}$ Vijaya Shetty S \\ ${ }^{1}$ Supercomputer Education and Research Centre, Indian Institute of Science, Bangalore, India; \\ ${ }^{2}$ Department of Computer Science and Engineering, Nitte Meenakshi Institute of Technology, Bangalore, \\ India; \\ bhaktha@serc.iisc.ernet.in; vishruthvipra@gmail.com; likith.april13@gmail.com; \\ deepakb2994@gmail.com; kaushalm25@gmail.com; vijus_shetty@yahoo.com
}

\begin{abstract}
The impact of increasing urbanization has set an alarming concern of the consequences of loss of human lives in the event of a natural disaster such as floods. It is essential that disasters are detected at inception so that the severity of damage could be reduced by a greater extent. The readings received from the sensor at regular intervals are compared with the threshold and any increase is immediately updated to the user on the app. A graphical analysis indicates to the user the possible fluctuations of the parameters used for an appropriate inference of the situation at that instant. This system would be useful in detecting increasing water level, UV radiation, atmospheric pressure, temperature, humidity, and keeping track of the situation real time monitoring the probability of an occurrence of disaster. The application could be deployed near traffic subways or underpasses that have stagnant water due to a heavy rainfall to divert traffic thereby reducing traffic snarls at major parts of the city. A common man having access to internet can visit the website created or who possesses a Smartphone can download the application thereby benefiting from the system. Another main objective of the system developed is data collection. New datasets can be developed which help in better predicting similar disaster situations in the future by sensing common patterns overtime. Several kinds of datasets can be generated as per the application. These datasets will prove a tool against better disaster management for the times to come.
\end{abstract}

Keywords: Urbanization, UV Radiation, GPRS, Modular Sensors, Proximity Alert, Early Warning System and Disaster Management

\section{Introduction}

The integrated hardware device is set up in a particular environment [1]. The system consists of an array of sensors like Ultrasonic sensor, Moisture sensor, Humidity and Temperature sensor, UV sensor, Barometer and a GPRS shield for effective communication and to upload the data to the website so that the same can be reflected on the Android application. The hardware can be set up in an underpass, at sea shores, in the extremities of the farm land, in dams, rivers, lakes and other such water bodies and the stationary system thus installed, when powered by a power source, starts transmitting data readings from the sensors at regular intervals of time. The data that is being sent from the set up consisting of the array 
of sensors and the GPRS module is also transmitted to the website which has developed in this regard and according to the values of the metrics obtained from the sensors such as humidity, temperature, water level, percentage of content of moisture level in the soil and so on, appropriate notifications, alert messages are displayed on the location page of the website for better understanding of the situation [2]. A map of the user's current location is also shown on the website so that the user gets a clear indication as to where he is and how far he is from the region in concern. The data on the website is then broadcasted on the Smartphone application to the users. The users who install this particular application on their Smartphone and enable the phone to access their location will be able to see their current location and a marker showing the state of the sensor which is depicted by the colour of the marker. The user can also visit the website for more precise viewing experience. The user gets a notification asking him to be alert when the parameters measured exceed the threshold provided that the user enters the proximity of the affected region.

\section{Working Ideas}

There are in general several types of warnings based on the type of disaster that may occur in the real world. A few of them are contemplated below.

\subsection{Warning for floods}

The rain sensor is initiated on spells of rain. The ultrasonic sensor is triggered as a result and the data indicating the temperature, humidity and the water level is pushed to the database. Every deployment (at a particular geographical location) has a different threshold value. The water level values in the database are continuously compared with the critical threshold set to that particular sensor ID. Appropriate inferences are displayed after comparison. The rain sensor controls the frequency of data reads. Computation is done based on the signals received from the GPRS module. The computation consists of keeping track, which sensor is transmitting signals with the help of sensor ID and then sends the data to the Smartphone application. The Smartphone application displays a map where a street marker turns red in case of a flood like situation. It also displays temperature and humidity of the location.

\subsection{Warning for ultraviolet radiation}

As the depletion in the ozone layer has increased, the Ultraviolet radiations are entering our atmosphere at an ever increasing rate and are altering the health of people all over the world. The Ultraviolet Index is the measurement of the strength of the UV radiations. When exposed to excessive levels of radiation, it leads to harmful forms of Melanoma (Skin Cancer).

There are a number of Weather Applications which predict the extent of UV Radiations which are entering the Atmosphere and display it as an average value. In our system, we can install an Ultraviolet Sensor and get a precise reading of the local UV level reading and give notifications to the users when it exceeds the safety value. The data is reflected on the Website and Application and the user can act in accordance to the received readings as in [3].

\subsection{Warning for cyclones}

A barometer is a scientific device which is used to measure the atmospheric pressure. When the atmospheric pressure rises, it means that the weather will be dry. When the pressure falls, it means that wet and stormy conditions will persist and also may lead to cyclones. 
R Bhakthavathsalam, K Vishruth, Likith Ponnanna P B, Deepak B, M Kaushal and Vijaya Shetty S; An Intelligent

Wireless Modular System for Effective Disaster Management, Transactions on Networks and Communications, Volume 4 No. 3, June (2016); pp: 22-33

A barometer can be installed into the system to measure readings at constant intervals of time and uploaded to the Website and the Application and the latest value can be reflected on the same.

Appropriate notifications can be displayed on the Website as well as Application according to the readings of the barometer and the user can be warned in case of a low pressure situation [4].

\section{Algorithm of the Whole Working}

The algorithm given below depicts the entire flow of how the system works.

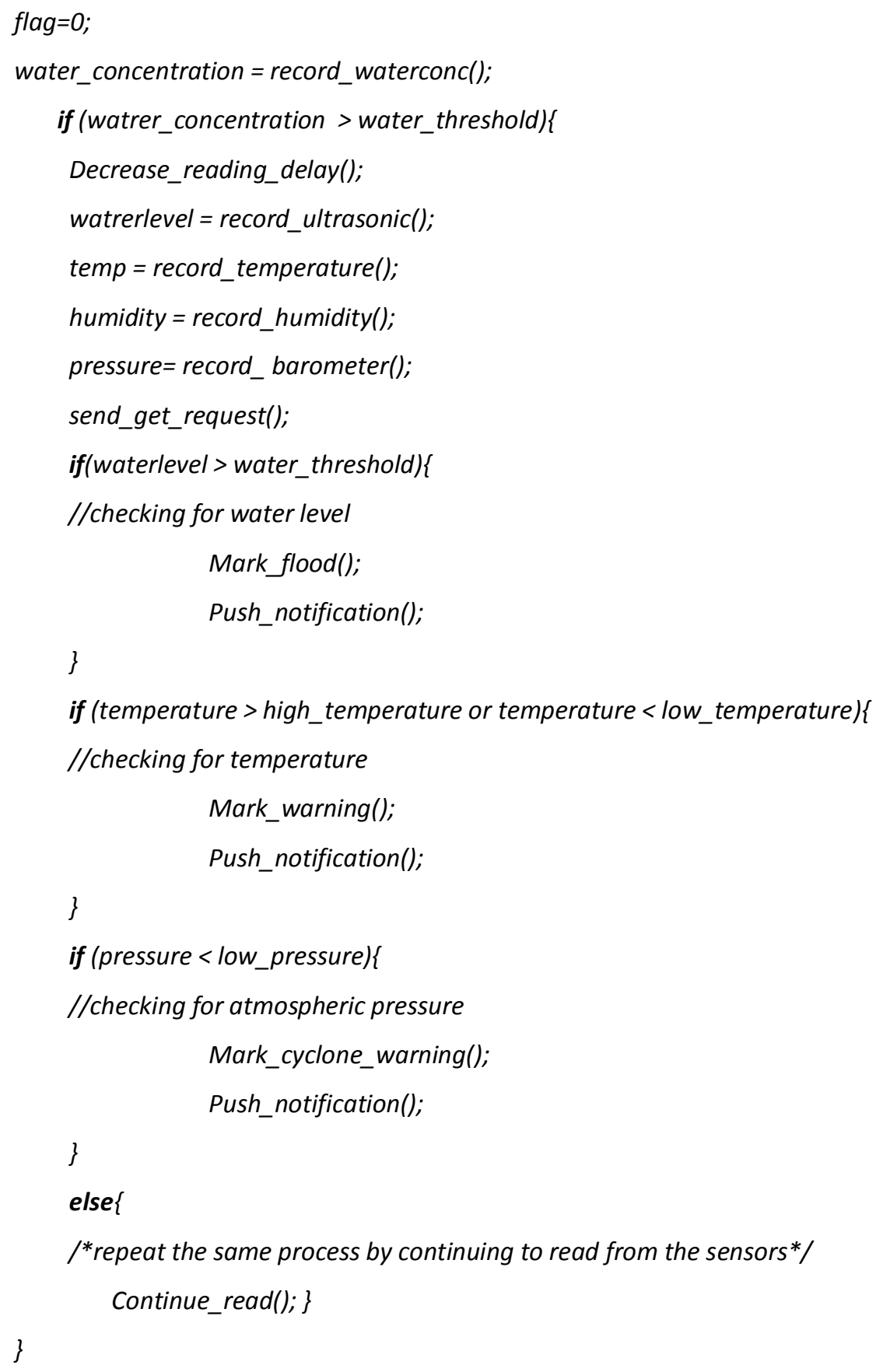




\subsection{Work-Flow}

The rain sensor detects the rain and triggers the ultrasonic sensor for faster recording of the parameters to be read. The ultrasonic sensor emits ultrasonic waves towards the ground and waits for the waves to return after bouncing back from the terrain. The time delay between the emissions of the sound waves to the receiving of the waves is recorded. By the relation: Distance = Speed * Time; the height up to which the waves have travelled is calculated. This height divided by two gives the exact height the waves travelled before bouncing back from the terrain thereby indicating the distance between the sensor and the ground. As the water level increases, the distance between the ground and the sensor decreases as the time taken for emission and receiving of the waves decreases. The humidity and temperature sensor records the humidity and temperature respectively of the surroundings of the sensor. The barometer and UV sensor record the air pressure and the ultraviolet radiation respectively. The recorded parameters are thus sent to the database for storage with the help of the GSM module by the microcontroller [5]. The database created is in the form of MySQL. The values are stored in the database by the phpMyAdmin which are accessed by the website and the Smartphone application. The application displays red markers in the region where the sensor was installed in the case of a flood, cyclone or lethal levels of ultraviolet radiation. A periodic view of the water levels, air pressure and the radiation from the sun can be seen on the website with the help of a graph which is developed using JavaScript. A notification is sent to the user in case the user is entering the danger zone to alert him as shown in Figure I.

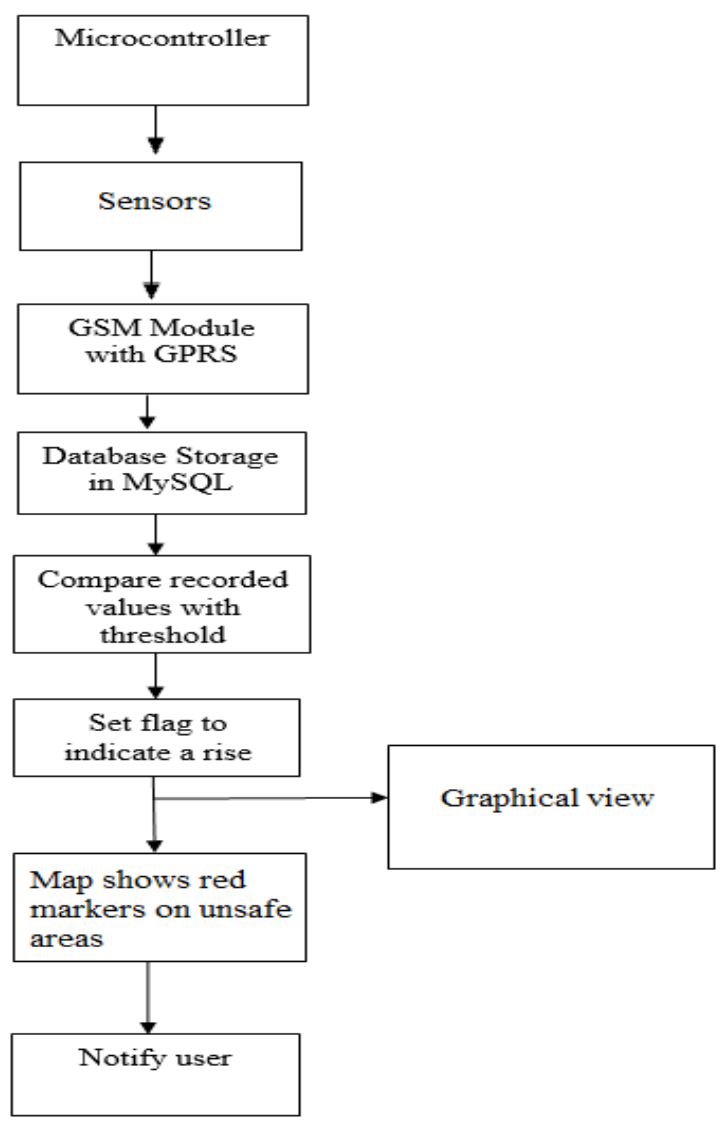

Figure 1: Working of the system

There are 2 main parts of the system, website and Smartphone application. 
R Bhakthavathsalam, K Vishruth, Likith Ponnanna P B, Deepak B, M Kaushal and Vijaya Shetty S; An Intelligent Wireless Modular System for Effective Disaster Management, Transactions on Networks and Communications, Volume 4 No. 3, June (2016); pp: 22-33

\subsubsection{Website}

There are 3 main webpages in the websites namely, home webpage, location specific webpage and contact.

Home page is the webpage which is linked to the Smartphone application. It links to all other webpages in the website. The user can select the location he wants to view from the drop down menu of "Select Location". A new webpage is opened with respect to the location chosen by the user. It also has provision for the user to know more about the website by clicking on the "About" button.

Location specific webpage contains a message about the condition of the place where the sensor has been installed is displayed. The messages thus displayed, depend on the values of the humidity, temperature and the water level in the vicinity of the sensor. The top most layer contains the summary of the environment of the sensor. The second layer displays the tuples of the database. The final layer displays the tuple values drawn from the database in accordance to the second layer. Below these messages, a map is displayed showing the location of the sensor placed. The entire system gets refreshed for every seven seconds. On clicking the button, a graph is displayed with time being the $\mathrm{x}$-axis and water level being the $y$-axis as shown in Figure II. The graph is coded with the help of JavaScript.

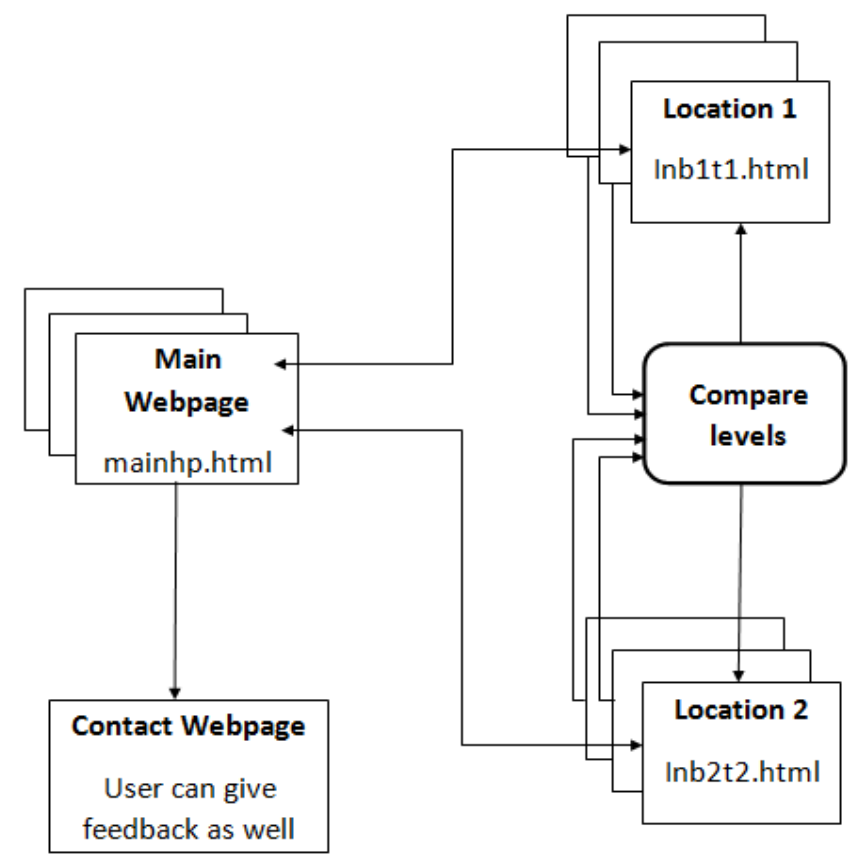

Figure 2: Working of the website

\subsubsection{Smartphone application}

The application can be divided into four main parts, main screen, sub screens, proximity receiver and web view.

Main Screen is the main activity screen that the application displays. It is the activity that the application displays when the application is opened by the user. The activity contains all the APIs provided by the google maps to display the map fragment on the screen. Markers are set on the map where the sensors 
are kept using the coordinates on the map fragment. The map also displays the location of the user once the user has activated the location services on his android phone. A zoom-in button is provided at the top right of the map for the user to zoom in to where he is standing. The map is downloaded when the internet services are available such that the application can be viewed even when there is no internet once the map is downloaded. The location services shall not be enabled on the downloaded map.

The markers on the map change colour based on the water level of the location. A red marker indicates that the location where the sensor is situated is flooded while a green marker indicates that the location is free of flood at that moment. The parameters from the sub screen linked to this screen are loaded into this activity for the comparison of the threshold of the water level. A radius is defined keeping the sensor coordinates as the centre and the alerts are broadcasted within the application when a user crosses the border of the radius towards the flooded region using the algorithm as follows:

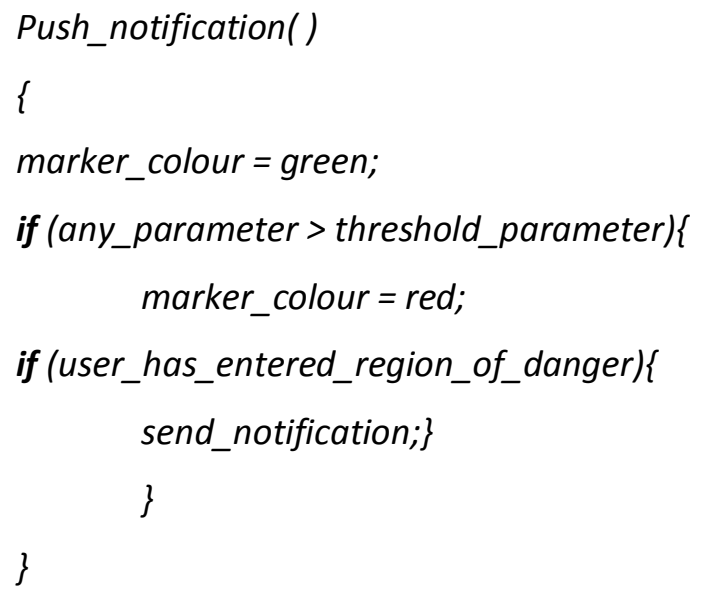

The alerts broadcasted are sent to proximity receiver class from here. A hyperlink is provided at the bottom of the screen for the user to access the website created.

Sub Screens are those which give the user details about the location the user has chosen. It contains a sensor ID, water level (in $\mathrm{cm}$ ), temperature (in ${ }^{\circ} \mathrm{C}$ ), humidity (in \%), air pressure (in $\mathrm{kPa}$ ) and ultraviolet radiation (in). the water level parameter displays the latest water level value pushed by the ultrasonic sensor into the database as shown in Table I.

The temperature displays the latest temperature value pushed by the temperature sensor into the database. The humidity parameter displays the latest humidity value pushed by the humidity and rain sensor into the database. The air pressure parameter displays the air pressure detected by the barometer and the ultraviolet radiation parameter gives the radiation exposed to the ultraviolet sensor.

Proximity Alert class is used to receive broadcasts sent by activities in the application. It detects whether the user has entered a specially marked area of concern and helps in producing notifications. Each notification has a unique notification ID, notification heading and the notification description. The notification is fired only if the region within the sensor's radius is in danger.

Web View class is used helps the user to navigate into the website providing a mobile based view.

The complete layout for the Smartphone application is illustrated as shown in Figure III. 


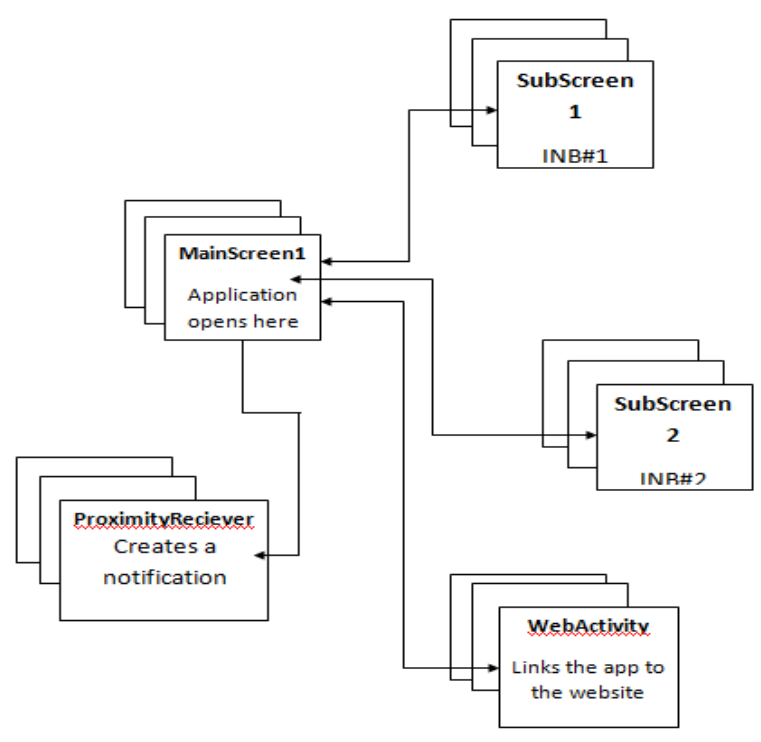

Figure 3: Working of Smartphone application

\section{Applications}

\subsection{Rivers and lakes}

In rivers and lakes, the sensor will be attached to a pole in the bank or any other fixed structure which will help the sensor stay above the water surface [6]. If the water level goes beyond the threshold level, then the system determines that the flood has occurred and it triggers warnings in both the website and the application triggers warning notification to the users in the proximity.

\subsection{Dams and reservoirs}

As the system can be adapted to various situations another application is monitoring the dams and reservoirs for the water level. As all the dams have a critical level of water that can be achieved and above which the dams structure will be severely compromised. In these situations, the system can trigger warnings to the person controlling the gates or automate the system to trigger the gate when the water raises above the critical value. This can help save several lives and also avoid large calamities.

\subsection{Supercomputing chambers}

In supercomputer labs, where the systems are very temperature and human sensitive, most labs now days are manually monitored and is prone to human error. The human monitoring can be completely removed and the system can monitor the required parameters and if they fluctuate by a large extent the person in charge can be alerted and avoid damages to expensive components.

\subsection{Underpasses and underground metros}

There have been several instances where flooding of underpasses and Underground metros have flooded and brought the traffic and the public transportation to a standstill. Applying this system in such situations can help in diverting the traffic to other areas as well as bringing the metro system to a halt when the raising water levels are detected in the underground tunnels and making for a better handling of the disastrous situation [7]. 


\subsection{Underground storages and labs}

A lot of highly secret labs are underground to avoid detection from satellite imagery and storages such as weapon of the military are stored in underground bunkers. These establishments are prone to flooding as they are in very low lying areas. This increase the risk of massive proportion of either leaking of sensitive data or damage to the equipment's stores. If the system is implemented in these situations it can monitor these floods if ever occurred and in other times they can help in monitoring the room's temperature and humidity and determining if the situations are conducive.

\subsection{Agricultural farms}

There have been instances where a farmer loses his crops because of lack of information about the atmospheric conditions to provide proper irrigation to the crops or sometimes there has been excessive water logging in fields and the crops have been destroyed. This can be avoided by installing the system in such places. This provides the farmer with required information about the water excessiveness or the shortage as a result he can plan his irrigation or drain water accordingly and also the system helps in monitoring the humidity, temperature and the air pressure using which the crops can be chosen or the necessary steps can be performed to bring the atmospheric conditions to the desired levels.

\subsection{Scientific data analysis}

The system can be a boon to the weather prediction organization as the system creates a large amount of data sets for different regions which when computed for patterns can reveal a lot of information which helps I predicting weather better in the future.

\subsection{Ozone layer depletion}

The UV radiation detection can help understand the quantity of Ozone in the atmosphere and can help people prevent from being exposed to the harmful radiation on a sunny day.

\section{Experiments and Results}

The experiments are conducted as given below:

- Initially, the system was setup in an ideal weather condition environment

$\circ$ Once the ideal conditions were tested the other test cases were performed by manual intervention.

- The results were then logged and the outputs were examined.

- The tests were performed on parameters including Water level, Temperature and Humidity

Case 1: Ideal scenario. That is, all the parameters are at within their ideal conditions.

Data is logged; no warning issued.

User is notified as SAFE.

Case 2: Water level is above the threshold.

Data is logged; warning is issued for users within the proximity of the sensors.

User is notified as ALERT - FLOOD!

Case 3: Water level is gradually increasing (above normal but below threshold).

Data is logged; no warning issued.

User is notified as SAFE, HOWEVER BE ALERT. 
Case 4: Temperature is-

a. Above $35{ }^{\circ} \mathrm{C}$ - User is notified as HOT.

b. Below $0^{\circ} \mathrm{C}$ - User is notified as FREEZING.

\subsection{Website snapshot}

The website shows the readings that are uploaded to the database in real-time. A front end graphical user interface is in place which shows the user the current readings as well as the appropriate warnings which is shown in Figure IV. It also shows the readings that are stored into the database taken according to the sensor id over a period of time to better ascertain the situation as shown in Figure V. The database shown in Table I represent the schema. Here the readings are stored according to their IDs for fetching them when required.

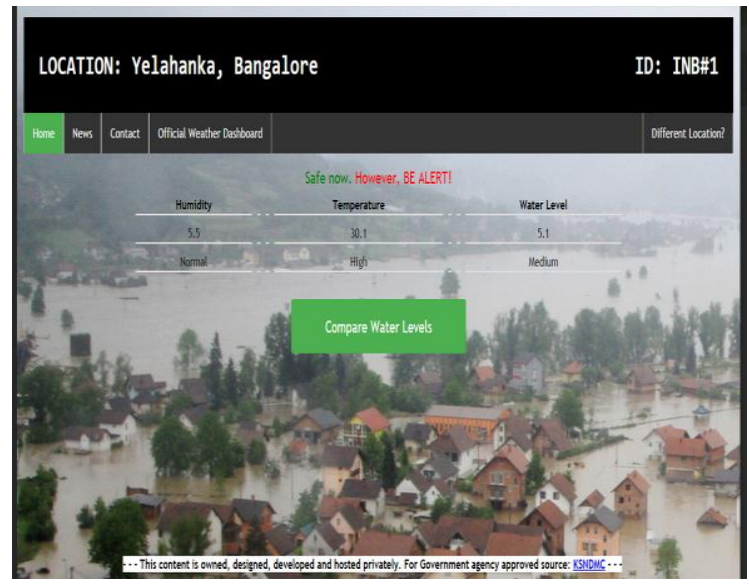

Figure 4: Location based values retrieved from database

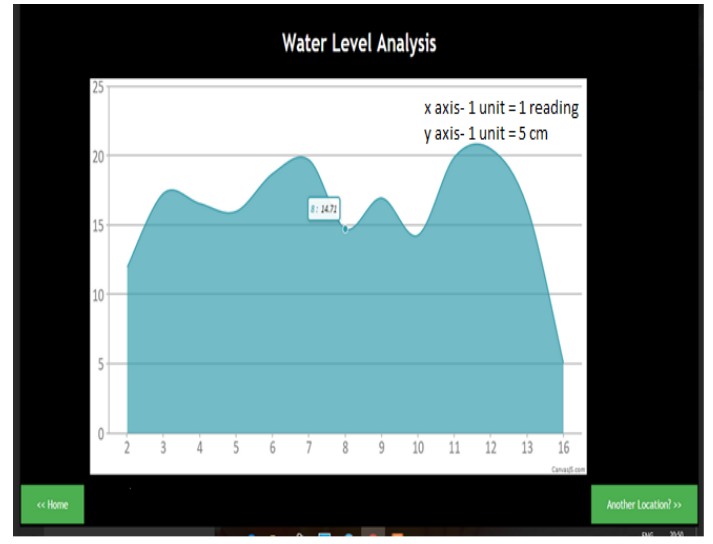

Figure 5: Graphical representation of the water level overtime

Table 1: Database schema for recording values

\begin{tabular}{|c|c|c|c|c|c|c|}
\hline $\begin{array}{c}\text { Reading } \\
\text { No. }\end{array}$ & Sensor ID & Temperature(ㅇ) & Humidity (\%) & $\begin{array}{c}\text { Water } \\
\text { Level(cm) }\end{array}$ & $\begin{array}{c}\text { Air Pressure } \\
\text { (bars) }\end{array}$ & UV level \\
\hline 1 & 1 & 28 & 54 & 0 & 1.01 & 9 \\
\hline 2 & 1 & 28 & 55 & 2 & 1.01 & 8 \\
\hline 3 & 2 & 29 & 55 & 12 & 1.01 & 4 \\
\hline
\end{tabular}

\subsection{Smartphone application snapshot}

The smartphone application has a map view which visually gives a bird's eye view of an area where the module installations are done and provides appropriate visual colour coded warnings to show the safe areas as shown in Figure VI. It also provides real-time readings of a particular location and displays them. This is represented in Figure VII. 


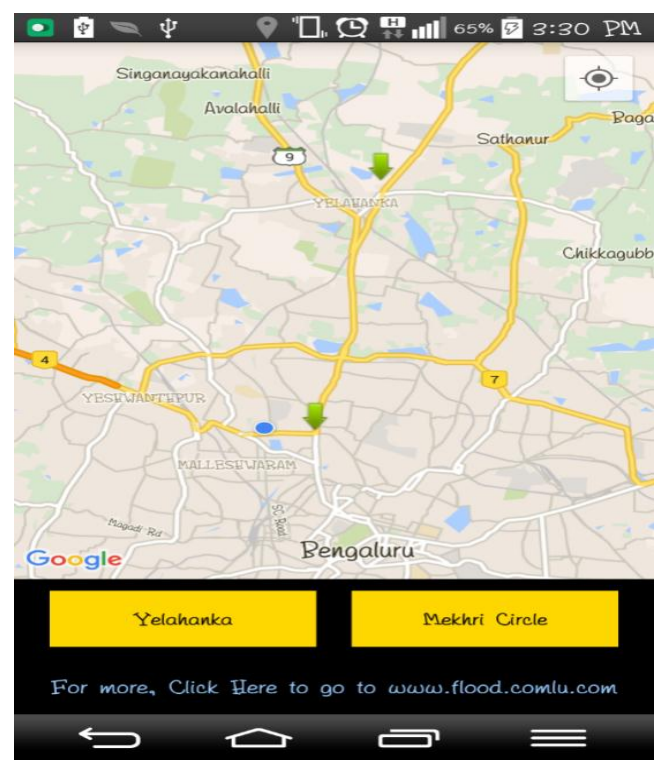

Figure 6: Green markers on the map showing safe locations

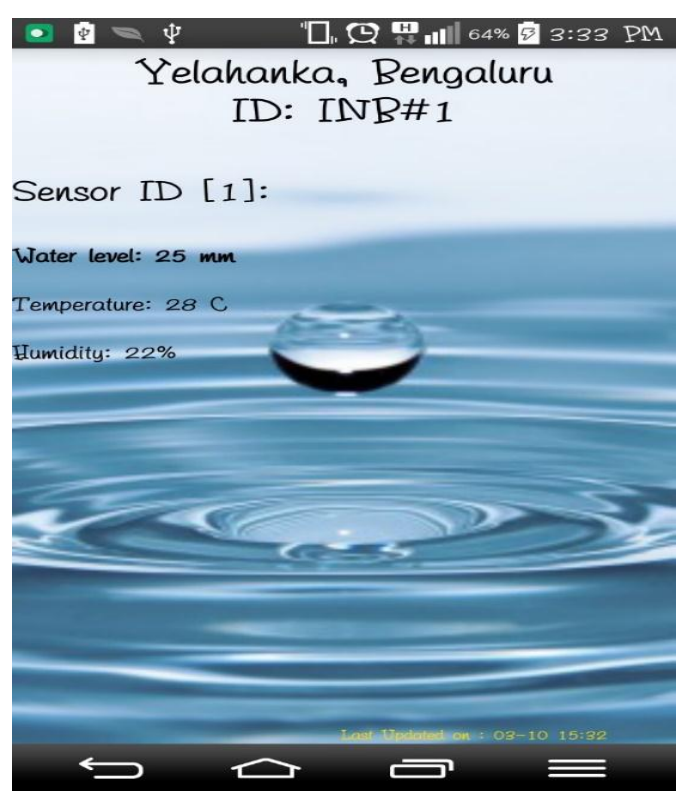

Figure 7: Sensor reading retrieved from a location

\section{Conclusion and Future Enhancement}

Given that loss of life due to natural disasters are likely to increase in future years with the increasing population, an increased attention to disaster prevention and mitigation strategies are of utmost importance. We have already experienced that early warning systems have been an effective mechanism for reducing the impact of disasters; however, they are not abundant and should be prioritized in less developed countries with large at-risk populations and high frequencies of natural disasters. It is key that messaging and targeted communication strategies accompany forewarnings so that the population comprehends the imminent risk and can retort appropriately. Many fatalities are associated with risktaking behaviours, thus messages to evade going into flood waters and to curtail risky activities in all stages of the event may be successful in reducing fatalities. Additional, better land use organization and regulation of development can mitigate impacts of disasters. Studies on the associations between flood damages, natural hazard characteristics, and societal and demographic vulnerability factors can aid in informing and prioritizing disaster prevention and mitigation strategies. Finally, comparisons of the effectiveness of diverse policies and mitigation strategies would be able to inform future strategies and policy actions and ensure they are appropriate in specific contexts.

Data from the older quarters of the century put forward that floods have exacted a significant toll on the population when compared to other various natural disasters, mainly in terms of the size of affected populations. However, human defencelessness to and resulting floods is growing, in most parts due to population growth, urbanization, land use conversion, and climatic features associated with a growth in extreme shower events. In the future, the frequency and impact of floods on the population can be estimated to increase. Additional attention to preparedness and mitigation policies, predominantly in poorly developed regions, where the bulk of floods occur, and in Asia, an expanse disproportionately hit by deluges, can lessen the impact of future flood events. During worse situation, the system has to make wise judgement to support the victims in the swamped or flooded locations. The authorities would be clever to strategize according to the time and obtainable resources within for the rescue activities. In most cases the information on the water-logged locations are educated during critical level and this makes the 
R Bhakthavathsalam, K Vishruth, Likith Ponnanna P B, Deepak B, M Kaushal and Vijaya Shetty S; An Intelligent Wireless Modular System for Effective Disaster Management, Transactions on Networks and Communications, Volume 4 No. 3, June (2016); pp: 22-33

authorities to struggle in the rescue activities, eviler still if there are several localities to be monitored and wise actions to be made. When a system is proficient to alert the establishments as quick as the water rises, then they may react accordingly in ensuring the safety of the flood victims and every second count in the rescue efforts.

The goal of this system was to create a low-cost wireless high water detection system that senses rising water in real time and determines any potential flash floods [8]. The wireless high water detection system is built to identify rising water levels and to warn any potential flood risk. The system is cost effective and is suited for mass implementation irrespective of the geography. Apart from detecting an event of exceeding water level, the system also helps generate dataset over a period of time. With a live recorded dataset, the system could be further made 'smart' to make predictions with calculated risks and assumptions. The system could also be modified for customized water level analysis on the basis of area of installation and the height. The reading and update frequency could also be set depending on the requirement. The system is modular and could be linked to audio/visual display units making it useful for all sections of the community including people who do not use smartphones. As every installation of the system at a particular location is assigned a unique identification number, management in the mass implementation scenario could be much easier. System installations at a particular area (state wise, for example) could be grouped and all values from each of them could be populated in a single database making data management and consolidation much easier. The execution cost is invaluable to the efficiency and usefulness of the system towards mankind. The practicality of the system greatly helps to lessen overheads due to flooding and also prevents catastrophe at flood prone locations. A system for monitoring the flooding situation and alerting was developed especially for critical flood prone remote locations to guarantee mankind safety and savings to all the sectors. The flood alert information's can be displayed on LED display sign for the users plying on the motorway and for safety reasons could be placed

at strategic locations. Such information is real time and transmitted wirelessly from the measured location. The following are the future scope of this working system:

1. The modular system could be extended further by addition of sensors that detect soil moisture and this would be helpful to envisage crop cultivation fitness, etc.

2. Sensors could be added that evaluate air quality for monitoring pollution levels at an area.

3. System installed over a period of time could help generate a dataset which could be used effectively to analyse weather patterns and make intelligent predictions using variety of data mining techniques.

4. The system could also be linked to audio/visual devices like LED, buzzer, siren alarms for instant notification to people in the vicinity of the installation area.

\section{REFERENCES}

[1] Surface Drainage Monitoring and Flood Prediction using ZigBee Wireless Sensor Network for by Ka-Heng Chan, Wai-Wa Choi and Chi-Seng Cheang Faculty of Science and Technology, University of Macau, Macau SAR, China10.1109/ISANP.2014.7026695@2014 IEEE, INSPEC Accession Number 14885657. 
[2] Aggregation in Wireless Sensors for Flood Forecasting System and Intelligent Data Classification by El Mabrouk Marouane, Ezziyyani Mostafa, Essaaidi MohamedLaSIT Laboratory, Faculty of Sciences and Technologies, UAE,978-1-4799-7391-0/14/\$31.00 @2014 IEEE

[3] "WHO | Ultraviolet radiation and health"- http://www.who.int/uv/uv_and_health/en/

[4] “How Does a Barometer Measure Air Pressure?" - http://weather.about.com/od/weatherinstruments/a/ barometers.htm

[5] Detection of Malicious Nodes with Secure Sensor Network SUM Aggregation by Choi, S. ; Dept. of Comput. Sci., Purdue Univ., West Lafayettee, IN, USA ; Ghinita, G. ; Bertino, E. 978-1-4673-1564-7/12 C2012 IEEE INSPEC Accession Number:13272216

[6] Flood Monitoring using An autonomous and Adaptable Wireless Device by Plessi, V. ; Dept. of Electr. \& Comput. Eng., Missouri-Rolla Univ., Rolla, MO ; Bastianini, F. ; Sedigh-Ali, S. 0-7695-2655-1/06 (C 2006 IEEE INSPEC Accession Number: 9165507

[7] "The Monsoon and water logging in Indian cities" - http://www.waterandmegacities.org/the-monsoonand-water-logging-in-indian-cities/

[8] Flood level indicator and risk warning system for remote location monitoring using flood observatory system by Sivakumar Subramaniam, Vigneswara Rao Ganapathy, Sivarao subramonian and Abdul Hamid Hamidon 